$\mathbb{P}$ periodica polytechnica

Civil Engineering

$54 / 2(2010) 79+88$

doi: 10.3311/pp.ci.2010-2.02

web: http://www.pp.bme.hu/ci

(c) Periodica Polytechnica 2010

RESEARCH ARTICLE

\section{Combined shear and patch loading of girders with corrugated webs}

\author{
Balázs Kövesdi / Ulrike Kuhlmann / László Dunai
}

Received 2010-03-16, revised 2010-04-30, accepted 2010-07-05

\begin{abstract}
The corrugated steel plate has been increasingly used as web of steel and composite bridges. When such a bridge is incrementally launched, the web panels are often subjected to combined shear and patch loading. There are only a few investigations available in the literature which are dealing with this interaction type, therefore the current research of the authors focuses on the analysis of the combined shear and patch loading in case of corrugated web girders. Based on patch loading tests executed on 12 short span specimens, a numerical model is developed and verified. A numerical parametric study is conducted to analyse the structural behaviour and the interaction between shear and patch loading in the typical parameter ranges used in bridges. Based on the large number of numerical calculations a design equation for interaction of shear and patch loading is proposed.
\end{abstract}

\section{Keywords}

steel bridge $\cdot$ corrugated web · patch loading $\cdot$ shear resistance $\cdot$ shear and patch loading interaction

\section{Acknowledgement}

This work is connected to the scientific program of the "Development of quality-oriented and harmonized $R+D+I$ strategy and functional model at BME" project. This project is supported by the New Hungary Development Plan (Project ID: TÁMOP4.2.1/B-09/1/KMR-2010-0002).

Financial support is provided by the German Academic Exchange Service DAAD, and by the Hungarian National Scientific Research Foundation OTKA - T049305 project, which is gratefully acknowledged.

\section{Balázs Kövesdi}

Department of Structural Engineering, BME, H-1111 Budapest, Múegyetem rkp. 3-5., Hungary

e-mail: balazskovesdi@vbt.bme.hu

\section{Ulrike KuhImann}

Institute of Structural Design, University of Stuttgart, Pfaffenwaldring 7, 70569 Stuttgart, Germany

\section{László Dunai}

Department of Structural Engineering, BME, H-1111 Budapest, Múegyetem rkp. 3-5., Hungary

\section{Introduction}

In the practice during launching of a bridge structure when the launching device does not yet reach the next pier, large shear and transverse force can be introduced at the previous pier at the same time. This situation is shown in Fig. 1. In this case the corrugated web is loaded by large shear and transverse forces, so that the interaction should be considered in the design.

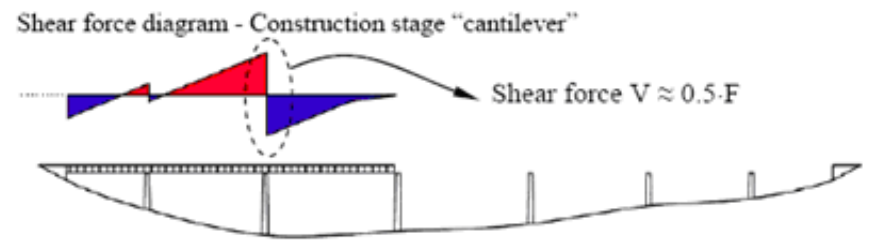

Fig. 1. Shear force distribution during launching, from [1].

In case of bridge structures, which have mostly high webs, the main part of the shear resistance comes from the contribution of the web. Bridges which are erected by incremental launching have mostly thick webs and long loading lengths at the launching device, therefore the main part of the patch loading resistance comes also from the web contribution. In case of corrugated web girders it is especially true because due to the corrugation profile the web contribution in the patch loading and in the shear resistance is increased. Due to the enlarged role of the web in both resistances the interaction of shear and patch loading of the corrugated web should be extensively investigated.

Interaction of bending moment and transverse force is also an important aspect of the load bearing capacity. Mainly the flanges are responsible to carry the large bending moments and at the same time flanges can have an important role in the patch loading resistance as well, especially in case of bridges where the flanges are relative thick. Therefore the interaction of these two effects, or the interaction of all three effects (bending moment, shear and transverse forces) should be also considered in the design. In the current research as a first step the interaction of shear and transverse force is investigated and presented in the current paper. The interaction with bending moment is the subject of future investigations.

The interaction behaviour of steel girders subjected to combi- 


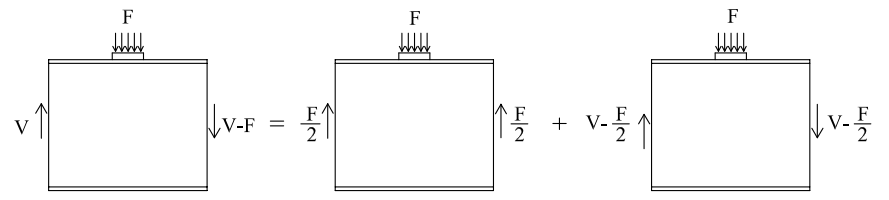

Fig. 2. Subdivision of the combined load into two basic load cases, from [1]

nation of shear and patch loading was studied only scarcely by researchers in the past. Even in case of flat web girders there are only a limited number of investigations available in the literature. No considerations of the interaction between shear and patch loading is made in standards neither for flat web nor for corrugated web girders. The current Eurocode 3 Part 1.5 [2] standard does not provide any rules for this interaction type.

Only one paper is found dealing with the shear and patch loading interaction of corrugated web girders [3]. No experiments are executed to analyse this interaction behaviour and only a limited number of numerical calculations are available for this topic. The previously executed calculations are focused on a narrow parameter range that is not typical for bridge structures. The aim of the paper is to study the interaction behaviour more throughly for a wider parameter range.

Patch loading experiments have been executed on 12 large scale test specimens at the Department of Structural Engineering of the Budapest University of Technology and Economics in 2008. Based on the experimental background numerical model is developed by the Finite Element Program Ansys 10.0 [4]. By the numerical simulation of all test specimens the developed model is verified. A comprehensive parametric study is conducted to analyse the interaction behaviour under combined shear and patch loading of corrugated web girders. The structural behaviour of the analyzed girders are investigated and the transition between the shear and patch loading failure modes are studied.

The interaction behaviour is investigated in a large parameter range. The analyzed parameters are the web depth, web thickness, the corrugation angle, fold length and the loading length. In total 370 numerical calculations are executed on girders with different geometries. Results are compared to the previously developed interaction curves and a new design interaction equation is proposed which is applicable for corrugated web girders in a large parameter range.

\section{Review of previous investigations}

\subsection{General}

All studies on the interaction between shear and patch loading have in common that the combined load is subdivided into two basic load cases ("pure patch loading" and "pure shear force"). Fig. 2] shows the separation methodology. The shear stresses due to "pure patch load" are already included in the patch loading resistance model and a reduction of the load carrying capacity is caused only by the additional shear stresses coming from "pure shear force".

\subsection{Previous investigations on corrugated web girders}

The corrugated steel plate is widely used as web of steel and composite bridges, therefore many literatures are focusing on the structural behaviour of the corrugated web girders. This paper focuses on the interaction of shear and transverse forces, therefore literatures which are dealing with patch loading or shear buckling or with the interaction of them are studied here.

Experimental research on the patch loading resistance of girders with trapezoidally corrugated webs started in 1987 by LeivaAravena and Edlund [5]. In 1988, Kähönen [6] made six tests on different girders, where the web crippling was analysed under patch loading. Elgaaly and Seshadri made five tests in 1997 [3], which were performed on a simply supported beam by varying the position of the applied load. Further numerical investigations were conducted by Luo and Edlund in 1996 [7]. All the previous tests and numerical calculations focused on typical building structures, where the load is transferred from the purlins to the roof girder with corrugated web, and the loading length was usually very short. In all cases, the failure was due to vertical bending of the flange and crippling of the web under the load. These numerical and experimental investigations were extended to the typical parameter range used in bridges by Kövesdi et al. [8]. Based on the large number of numerical and experimental results a modified design method is developed to determine the patch loading resistance of corrugated web girders.

There are also several papers dealing with the shear buckling resistance of corrugated web girders. Research in this topic was started by Easley and McFarland [9] in 1969, they proposed a global shear buckling equation for corrugated webs by treating the corrugated web as an orthotropic flat web. Experimental investigations were completed by Elgaaly et al. [10], Yamazaki [11], Bergfelt et al. [12], Driver et al. [13], Scheer [14], and Moon et al. [15] focusing on the determination of the shear buckling resistance of corrugated web girders. Numerical investigations were executed by Lou and Edlund in [16] and by Jongwon et al. [17] where the different geometric parameters (web depth $\left(h_{w}\right)$, span $(L)$, web thickness $\left(t_{w}\right)$, corrugation depth $\left(a_{3}\right)$, corrugation angle $(\alpha)$ and the fold length $\left(a_{1}\right.$ and $\left.a_{2}\right)$ were analyzed. (Notations are presented in Figs. 4 and 5.) Based on numerous experimental and numerical investigations, design methods were developed for all the tree types of shear buckling failure modes, namely for the local, global and interactive shear buckling.

Despite many papers available dealing with the patch loading and shear buckling resistance of corrugated web girders, there are only a limited number of investigations dealing with shear and patch loading interaction even for flat web girders and only one paper is available for corrugated web girders [3]. There are no experimental results available in this topic for corrugated web girders, and only results of 20 numerical calculations can be found in the literature. There are also no standard recom- 
mendations in Eurocode 3 Part 1-5 [2]. Elgaaly and Seshadri investigated the shear and patch loading interaction of corrugated web girders in 1997 [3]. The main aim of their research was the determination of the patch loading resistance of corrugated web girders. They conducted experiments on 5 test specimens and the pure patch loading resistances were determined. Based on the experiments a numerical model was developed and the pure patch loading resistance and the interaction between bending, shear and patch loading was studied in frame of a numerical research program. The studied parameter range was strongly limited and only 20 calculations are conducted to analyze the shear and patch load interaction. Based on the numerical calculations the interaction proposal of Eq. (1) was derived for corrugated web girders.

$$
\left(\frac{V-0.5 \cdot F}{V_{R}}\right)^{1.25}+\left(\frac{F}{F_{R}}\right)^{1.25} \leq 1.0
$$

Results of the numerical calculations and the recommended interaction curve according to Elgaaly [3] can be seen in Fig. 3 .

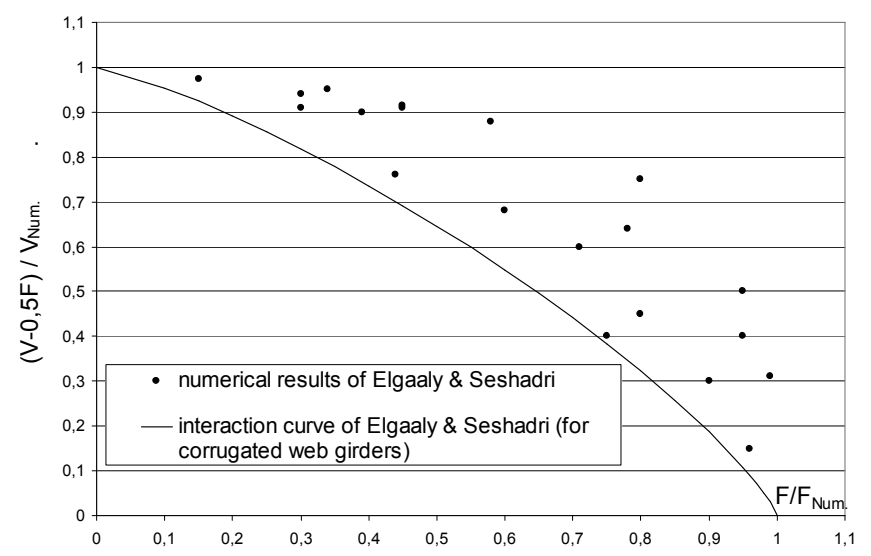

Fig. 3. Numerical results for corrugated web girders and proposed interaction curve.

The aim of the current investigation is to extend the analyzed parameter range. More corrugation profiles, longer loading lengths and a larger range of web and fold slenderness ratios are investigated. Based on the numerical calculations an interaction curve is developed which is based on an extended parameter range.

\section{Numerical investigations}

3.1 Experimental background and numerical model development

Experimental background of the numerical model development is the test program executed at the Budapest University of Technology and Economics in Hungary on 12 simply supported large scale test specimens. The test arrangement and geometry of the specimens can be seen in Fig. 4

The aim of the tests is to determine the pure patch loading resistance of corrugated web girders with different geometrical arrangements, loading lengths and load positions and to give verification background for a Finite Element model development. The analyzed parameters are the followings: loading length ( $s=90 \mathrm{~mm} ; 200 \mathrm{~mm} ; 380 \mathrm{~mm}$ ), loaded fold (inclined, parallel fold or corner area), flange thickness $\left(t_{f}=20 \mathrm{~mm} ; 30 \mathrm{~mm}\right)$, $\operatorname{span}(L=1140 \mathrm{~mm} ; 1500 \mathrm{~mm} ; 1875 \mathrm{~mm})$, load eccentricity in transverse direction.

All the test specimens have the following constant geometries. Web plates are made of 500x6 mm corrugated steel plates. The corrugation profile is the same for all test specimens, and the geometry is shown in Fig. 5. Fold lengths are $a_{1}=210 \mathrm{~mm}$, $a_{2}=212 \mathrm{~mm}$, the projection lengths of the inclined folds are $a_{3}=133 \mathrm{~mm}$ and $a_{4}=165 \mathrm{~mm}$. The corrugation angle is $39^{\circ}$. Flanges are made of $225 \mathrm{~mm}$ wide steel plates and the thickness is $20 \mathrm{~mm}$ for most of the specimens. In case of specimens \#5, \#6, \#9 and \#10 the flange thickness is $30 \mathrm{~mm}$. The span is $1875 \mathrm{~mm}$ if a parallel fold and $1500 \mathrm{~mm}$ if inclined fold is loaded. One test is made on a very short span girder, where the span is $1140 \mathrm{~mm}$.

The measured average yield strength and the ultimate strength of the flange material are $379 \mathrm{MPa}$ and $517 \mathrm{MPa}$, and for the web plate $373 \mathrm{MPa}$ and $542 \mathrm{MPa}$, respectively. The load is applied along the total width of the flange by a $50 \mathrm{~mm}$ thick plate. Three different loading lengths are used (90, 200 and $380 \mathrm{~mm}$ ). The effect of the loading eccentricity is also analyzed, therefore only a part of the flange along the width $(80 \mathrm{~mm})$ is loaded at first time by a centric load in transverse direction and in second case by a $30 \mathrm{~mm}$ eccentricity in case of specimens \#9 and \#10. In these two cases the load is applied by a $300 \times 80 \times 50 \mathrm{~mm}$ plate. The loading length is $300 \mathrm{~mm}$ and the load width is $80 \mathrm{~mm}$. The geometry for all specimens and the failure loads measured during the tests are summarized in Table 1 More detailed test program and results can be found in [18].

Tab. 1. Geometry of test specimens and patch loading resistances.

\begin{tabular}{ccccc}
\hline Specimen & $L[\mathrm{~mm}]$ & $s s[\mathrm{~mm}]$ & loaded fold & $F_{\text {exp }}[\mathrm{kN}]$ \\
\hline 1. & 1500 & 90 & inclined & 754.2 \\
\hline 2. & 1500 & 200 & inclined & 956.5 \\
\hline 3. & 1875 & 90 & parallel & 764.8 \\
\hline 4. & 1875 & 200 & parallel & 949.0 \\
\hline 5. & 1875 & 200 & parallel & 1192.0 \\
\hline 6. & 1500 & 200 & inclined & 1119.3 \\
\hline 7. & 1140 & 200 & parallel & 1077.7 \\
\hline 8. & 1500 & 380 & inclined & 1263.9 \\
\hline 9. & 1500 & 300.80 & inclined (centric loading) & 1220.5 \\
\hline 10. & 1500 & 300.80 & inclined (eccentric loading) & 1090.0 \\
\hline 11. & 1875 & 380 & parallel & 1281.0 \\
\hline 12. & 1500 & 90 & corner area & 772.4 \\
\hline
\end{tabular}

A numerical model has been developed for the test specimens using Finite Element software ANSYS v10.0 [4]. The analyzed girder is modelled using four node thin shell elements. 

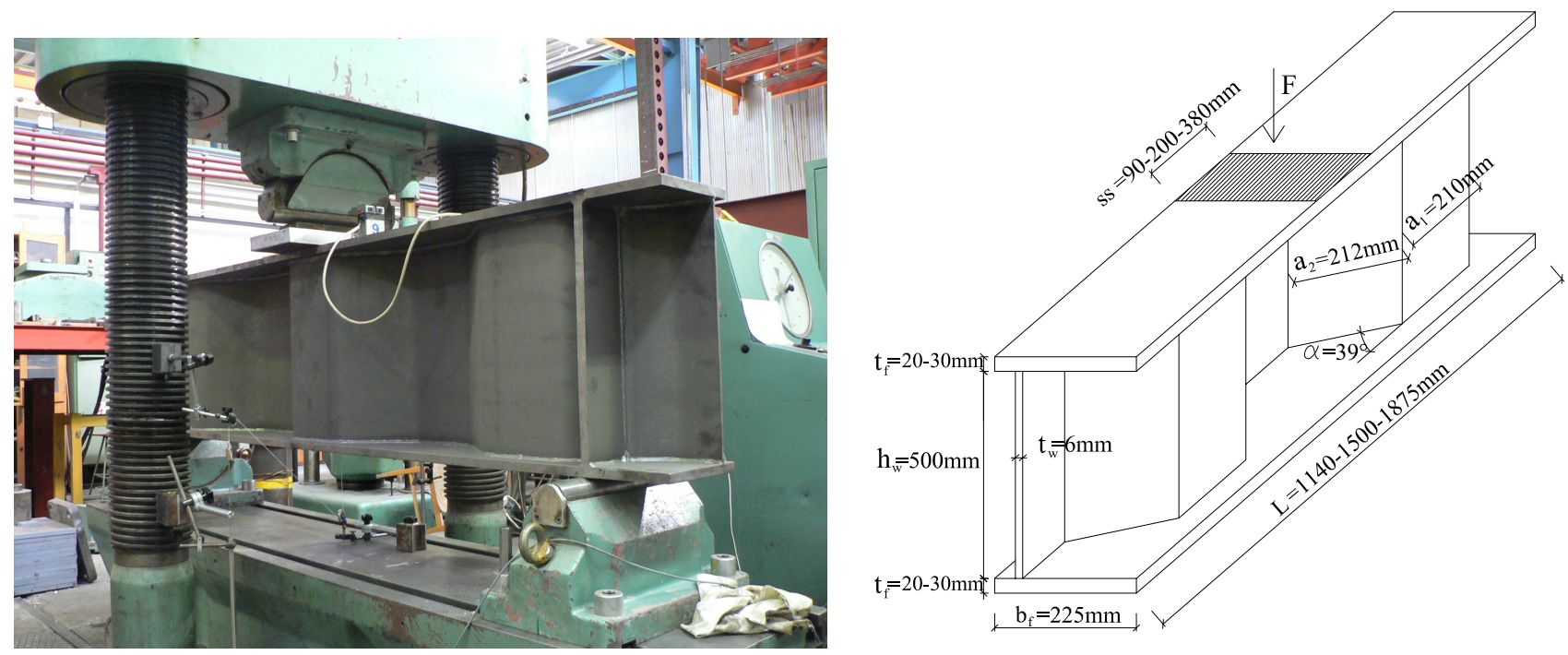

Fig. 4. Test specimen and test arrangement.

Fig. 6 6 shows the Finite Element mesh of the developed numerical model.

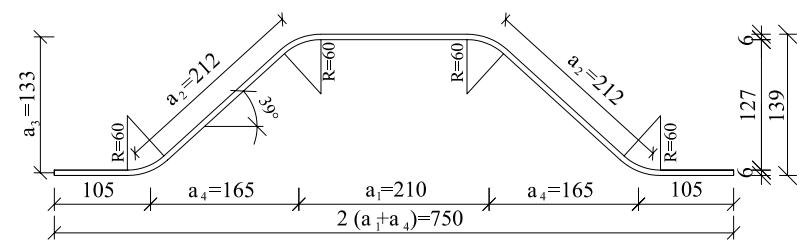

Fig. 5. Corrugation profile.

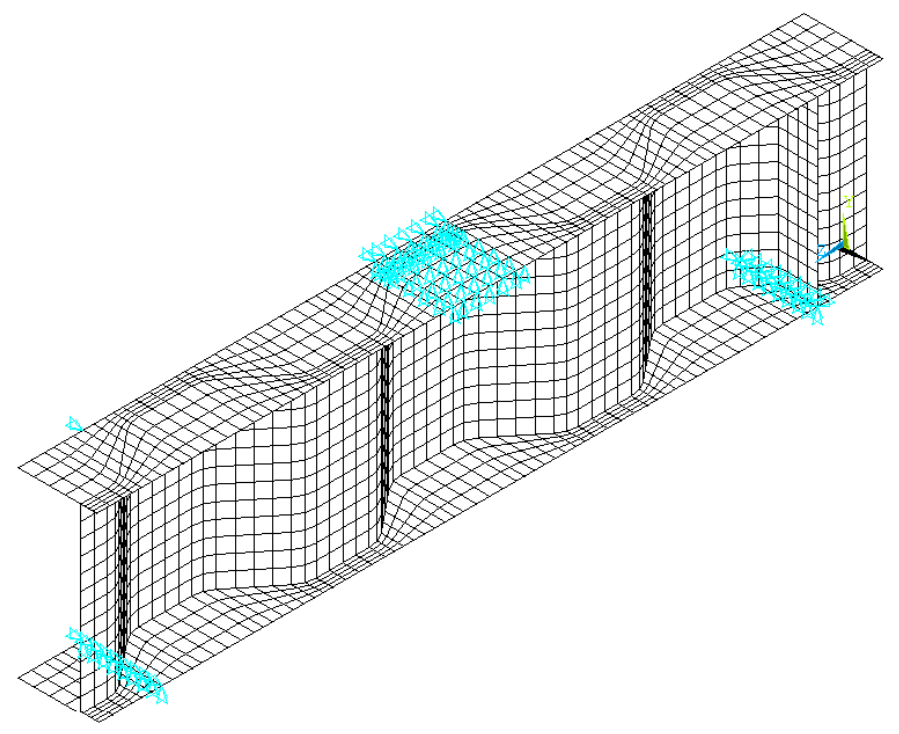

Fig. 6. Numerical model.

The applied material model is a linear elastic - hardening plastic model using a multilinear isotropic hardening rule with von Mises yield criterion. The material is linear elastic until reaching the measured yield strength with a Young modulus of $210000 \mathrm{MPa}$. The yield plateau is modelled to $1 \%$ strains without hardening. From the yield strength the material model is linear hardening with a reduced modulus until reaching the ultimate strength by $15 \%$ strains. The material is perfectly plastic when it reaches the ultimate tensile strength measured in the laboratory tests.

Ultimate loads are determined by geometrical and material nonlinear analysis using imperfections. The EN1993-1-5 [2] does not consist any recommendations for the equivalent geometric imperfections for corrugated web girders. An extensive investigation has been executed by Kövesdi and Dunai [18] to develop possible imperfection shapes and applicable imperfection magnitudes for patch loading of corrugated web girders. Four imperfection shapes are analysed in this paper. The first critical buckling mode, the ultimate shape and the sine wave imperfection form are investigated, and a modified sine wave imperfection shape is developed to predict the first buckling mode. Imperfection sensitivity is studied for all imperfection shape types and based on the executed experiments imperfection scaling factors are developed. Calculations showed that the applicable scaling factor is the fold length divided by 200 , if the first buckling mode or the modified sine wave shape is used as equivalent geometric imperfection. These investigations and conclusions are valid for patch loading of corrugated web girders.

Since no recommendations can be found in the literature dealing with the equivalent geometric imperfection form studying the shear buckling resistance of corrugated web girders, the imperfection shape applied in the nonlinear calculations is the first eigenmode in the current investigations. The applied magnitude is the fold length divided by 200 , if the model is loaded only by a transverse force. If the analyzed model is loaded by a shear force, the applied imperfection magnitude is the web height divided by 200 . In case of the combined loading situations the imperfection magnitude is calculated from these two values in the function of the ratio of the transverse and shear forces.

The Finite Element model exhibits the same structural behaviour and the same failure mode which have been observed during the tests. The measured patch loading resistances are 


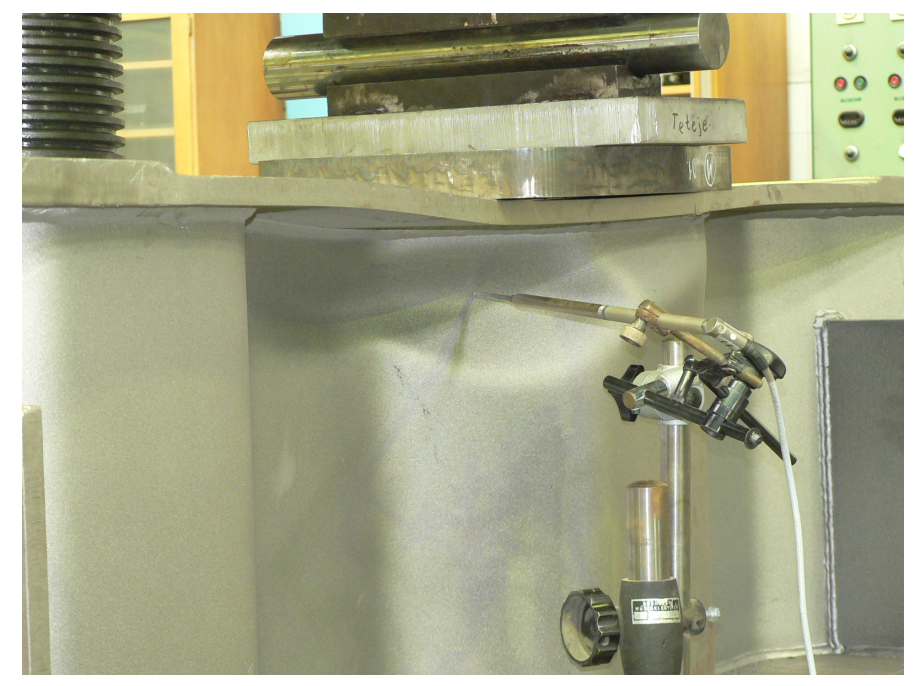

Fig. 7. Comparison of the experimental and numerical failure modes.

compared to the numerical calculations, the experimental failure modes to the numerical failure modes and the measured load displacement curves to the equilibrium paths of the numerical analyses. Fig. 7 shows the comparison of the experimental and numerical failure modes for one specimen. On the left side of Fig. 7 the observed experimental failure mode and on the right side the result of the finite element simulation is presented. The comparison proves that the buckling pattern of the numerical model is the same as the observed ultimate shapes in the experiments.

The measured and calculated load - displacement curves are also compared. The analysis showed a good agreement between the experimental and numerical results. The detailed model verification and model development is published in [18]. The load - deflection curves (curves (1) and (2)) and the load - lateral displacement curves (curves (3) and (4)) are presented in case of one specimen in Fig. 8. Deflection of the specimens is measured at the mid-span on the lower flange and the lateral displacement is measured on the web plate about $100 \mathrm{~mm}$ below the upper flange under the load introduction place, as shown in Fig. 7.

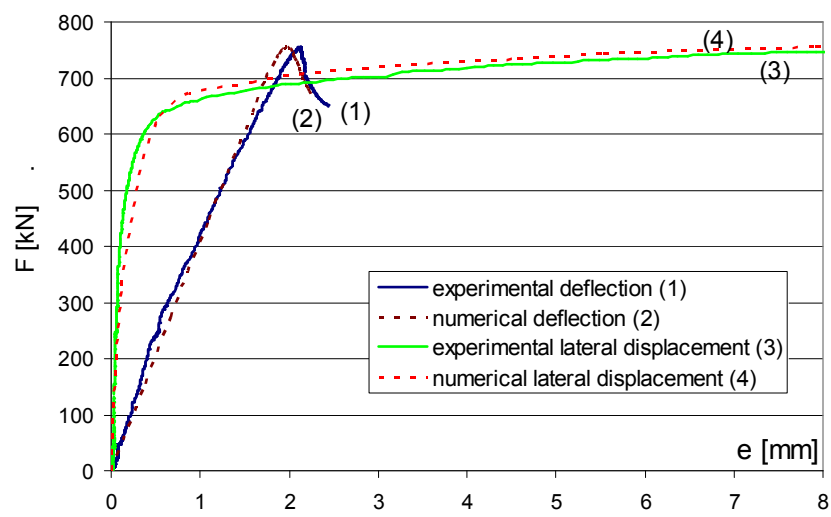

Fig. 8. Experimental and numerical load - displacement curves.

The studied phenomenon is characterised by a limit-point instability behaviour. The limit point (highest point) of the loaddeflection diagram represents the ultimate load of the speci-

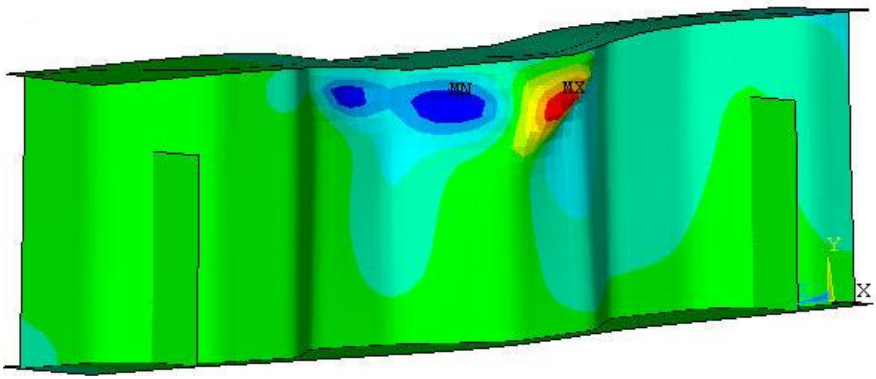

mens.

Tab. 2. Comparison of the measured and calculated results.

\begin{tabular}{cccc}
\hline Specimen & $F_{\text {exp }}[\mathrm{kN}]$ & $F_{\text {num }}[\mathrm{kN}]$ & difference $[\%]$ \\
\hline 1. & 754.2 & 748.7 & 0.7 \\
\hline 2. & 956.5 & 915.7 & 4.3 \\
\hline 3. & 764.8 & 756.6 & 1.1 \\
\hline 4. & 949.0 & 941.9 & 0.8 \\
\hline 5. & 1192.0 & 1131.1 & 5.1 \\
\hline 6. & 1119.3 & 1079.2 & 3.6 \\
\hline 7. & 1077.7 & 1047.7 & 2.8 \\
\hline 8. & 1263.9 & 1239.8 & 1.9 \\
\hline 9. & 1220.5 & 1193.2 & 2.2 \\
\hline 10. & 1090.0 & 1074.8 & 1.4 \\
\hline 11. & 1281.0 & 1245.3 & 2.8 \\
\hline 12. & 772.4 & 732.1 & 5.2 \\
\hline
\end{tabular}

Comparison of the measured and calculated ultimate loads show a good agreement between test results and numerical simulations. Differences in the patch loading resistance are in all cases under $6 \%$. The comparison is summarized in Table 2

\subsection{Modelling of the shear and patch loading interaction behaviour}

Panels loaded with combined patch load and shear force can be classified into 4 classes according to [1]. The schematic overview of the subdivision is illustrated in Fig. 9 . Basis of the classification is the different $V_{1} / V_{2}$ ratio.

Case "a" shows the pure patch loading situation if the girder is loaded along a small part of the flange. In this case $V_{1}$ and $V_{2}$ forces are equal, and the $V_{1} / V_{2}$ ratio is equal to -1 .

Case " $b$ " corresponds to the patch loading situation with an interacting shear force. The dominant effect is the patch load and 
the shear force makes the $V_{1}$ and $V_{2}$ reaction force distribution asymmetrical. The ratio of $V_{1} / V_{2}$ varies between 0 and -1 .

Case "c" represents the loading situation if the shear force is dominant and an interacting patch loading is also applied on the structure. In this case the shear force diagram does not change sign and the ratio of the $V_{1}$ and $V_{2}$ changes between 0 and 1 .

Case "d" shows the situation of pure shear without patch loading. The ratio of the $V_{1} / V_{2}$ forces is equal to 1 .

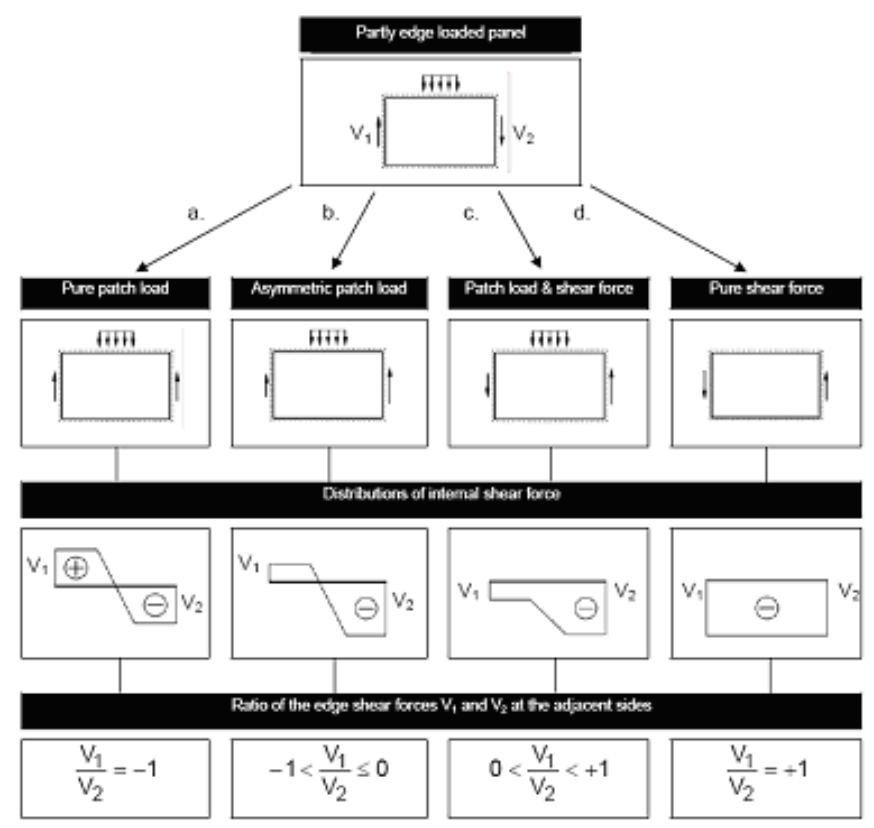

Fig. 9. Subdivision of panels loaded with combined shear and patch load, from [1].

This classification does not mean 4 independent classes of the structural behaviour of the loaded panel. In the first class (pure patch loading) failure mode is web crippling. In class " $d$ " (pure shear force) failure mode is shear buckling of the web. In the interaction zone there is a uniform transition between these two failure modes. This subdivision suggests, that it is sufficient, if only half of the girder (only one web panel) is modeled (Fig. 10. The applicability of this simplification is proved for the analyzed girders. This simplification is practical because the analyzed model is only the half of the whole one, therefore calculation time is significantly reduced. The second advantage is that both applied forces ( $V_{1}$ shear force and $F$ patch loading force) are input data of the model. $V_{1}$ and $F$ are the applied forces on the girder and $V_{2}$ might be calculated as a vectorial sum of these two forces, therefore the $V_{1} / V_{2}$ ratio can be controlled easily during the calculations. Defining $V_{1}=x \cdot F$ the numerical parametric study can totally be automatized. By changing the value of $\mathrm{x}$ the whole interaction domain $\left(-1<V_{1} / V_{2}<1\right)$ can be analyzed step by step. Based on the classification in Fig. 10 it can be stated that in case of bridge launching the asymmetric patch loading (case "b") has the most frequent occurrence, therefore this parameter range is analyzed with special regard.

Due to the model simplification and due to defining a plane of symmetry at the midspan a new model verification is needed. In case of pure shear force the analyzed loading situation is symmetrical therefore the definition of the symmetric plane is correct. If patch load is also applied the loading situation is asymmetrical and the equivalence of the calculation results made on the total and on the half girder has to be proved. Model verification is executed through the comparison of the load bearing capacities calculated on the full and on the half girder under several loading situations. Comparison is made for more girder geometries, too and on two levels: ultimate loads and failure modes are compared. Calculated ultimate loads are presented in Table 3 for one example. Definition and determination method of the ultimate load is the same as described in Section 3.1. The results show that only a minimal difference is between the two calculations $(0.34 \%)$. It proves that the plane of symmetry can be applied in case of the unsymmetric loading situation as well, because the plane of symmetry has no influence on the structural behaviour and on the ultimate loads in case of combined shear and patch loading. The reason of it is that the patch loading failure is a local failure what is not influenced by the global girder geometry. In case of shear buckling the applied load is symmetrical therefore the plane of symmetry is correct and does not indicate changes in the structural behaviour.

Tab. 3. Comparison of the ultimate loads.

\begin{tabular}{lcc}
\hline & $F_{\text {Ansys }}[\mathrm{kN}]$ & difference \\
\hline Full girder modelled & 2451.35 & \multirow{2}{*}{$0.34 \%$} \\
\cline { 1 - 2 } Half girder modelled & 2459.77 & \\
\hline
\end{tabular}

Failure modes are compared and presented in Fig. 11. The displacement vector sums are indicated on these Figures in [mm] units. It can be seen that the ultimate shapes and failure modes are the same, and the plane of symmetry does not change the failure mode in the cases of the analyzed models. The results prove that both models show the same structural behaviour and failure load. It means that this modelling simplification can be used to analyze the interaction of shear and patch loading of corrugated web girders.

\subsection{Analyzed parameter range}

Aim of the investigation is to extend the analyzed parameter range of Elgaaly et al. [3]. The investigated parameter ranges are the following (notations are given in Fig. 4):

- $h_{w} / t_{w}: 100-125-150-200-250$

- $a_{1} / t_{w}: 15-20-25-30-35$

- $\alpha: 20^{\circ}-30^{\circ}-40^{\circ}-60^{\circ}$

- $s s / h_{w}: 0.2-0.4-0.5-0.6-0.8$

\subsection{Structural behaviour}

To obtain the structural behaviour of the shear and patch loading interaction, different failure modes under pure patch loading, 

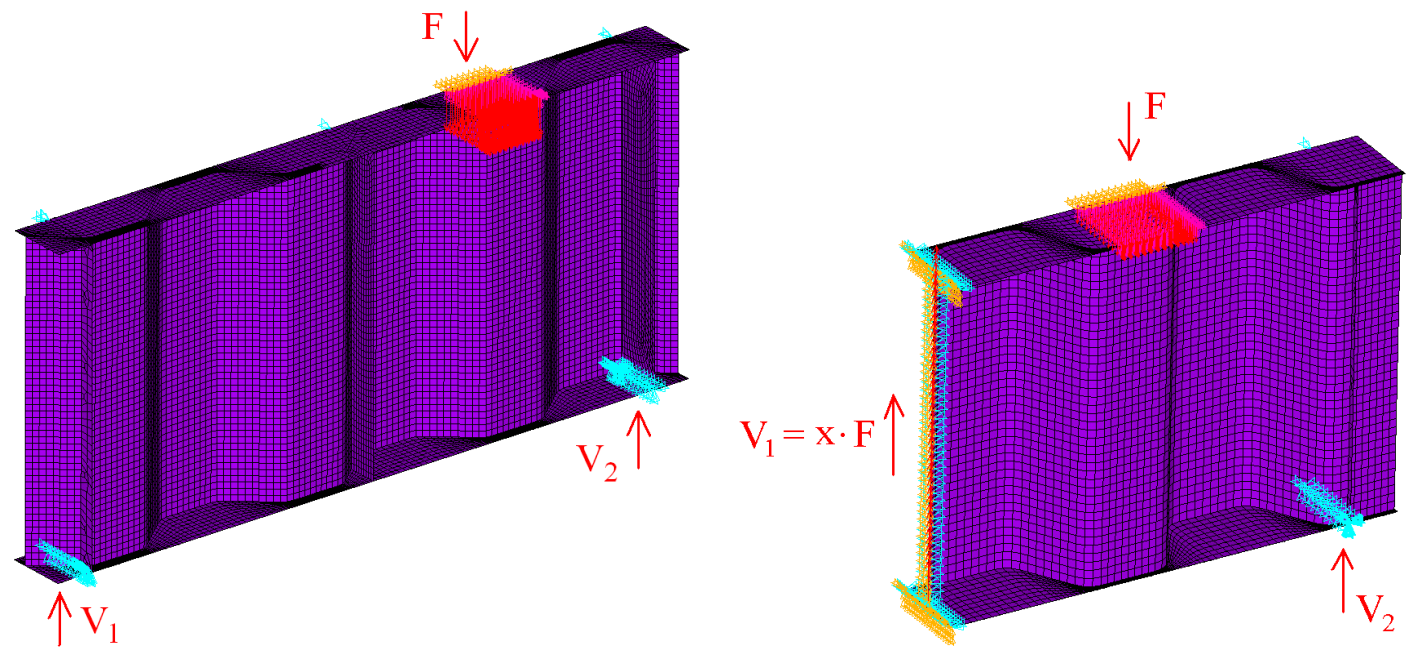

Fig. 10. Applied numerical models.
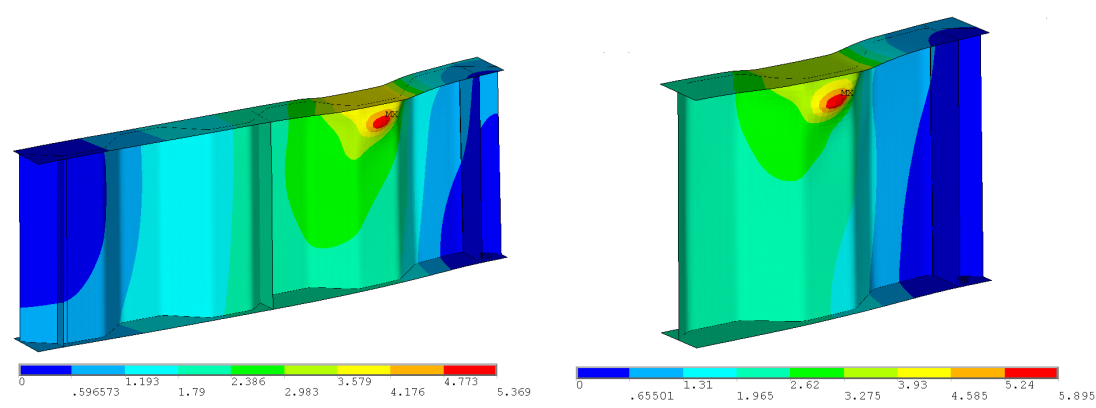

Fig. 11. Comparison of the ultimate shapes.
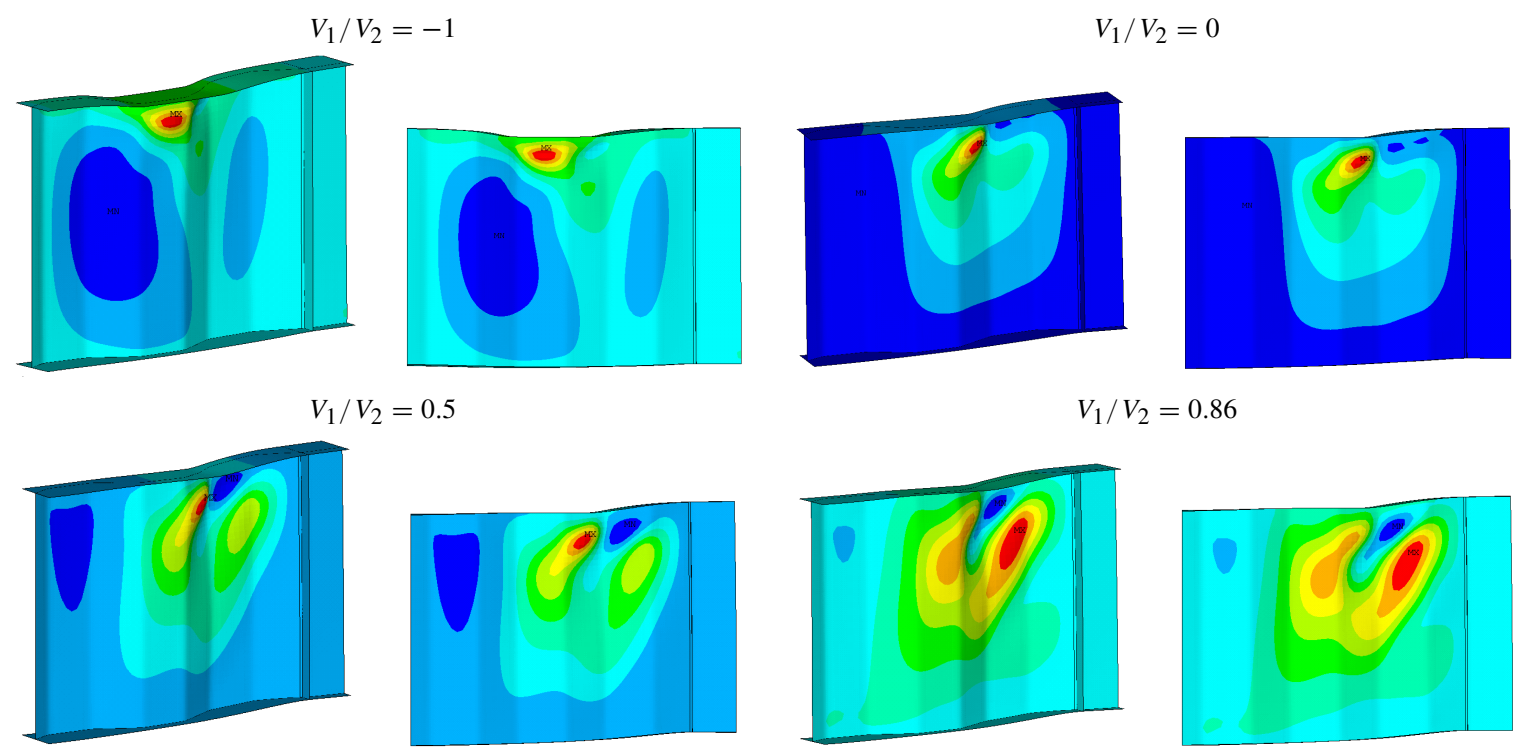

$V_{1} / V_{2}=1.0$
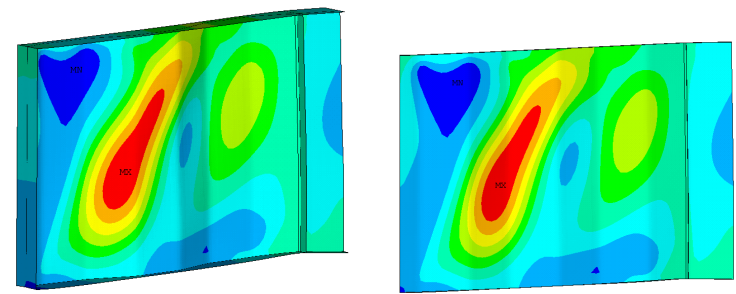

Fig. 12. Comparison of failure modes. 
pure shear force and combined loading are analyzed and compared to each other. The comparison of the lateral displacements are presented in Fig. 12

In the case of pure patch loading the failure mode is local web crippling (case $V_{1} / V_{2}=-1$ ), in the case of pure shear force the failure mode is shear buckling of the web (case $V_{1} / V_{2}=1$ ). In the interaction zone a uniform transition between the two failure modes can be observed, and no strict limit can be drawn between the two failure modes.

The lateral displacements in the loaded web folds are analyzed under different loading conditions. Fig. 13 shows the lateral displacements along the web depth in a parallel fold and Fig. 14 in an inclined fold. In the case of pure web crippling there is only one peak in the diagrams under the loaded flange $\left(V_{1} / V_{2}=-1\right)$. In the case of pure shear force $\left(V_{1} / V_{2}=1\right)$ the maximal lateral displacement is at the position of the tension band. In the interaction range coming from pure patch loading to the pure shear force the effect of the shear buckling is more dominant. In the case of asymmetric patch loading $\left(-1<V_{1} / V_{2} \leq 0\right)$ there are two peaks on the diagrams, one belongs to web crippling and one to the tension band of the shear buckling. In the case of $0<V_{1} / V_{2}<1.0$ the shear buckling is more dominant and only one peak can be seen on the diagrams.

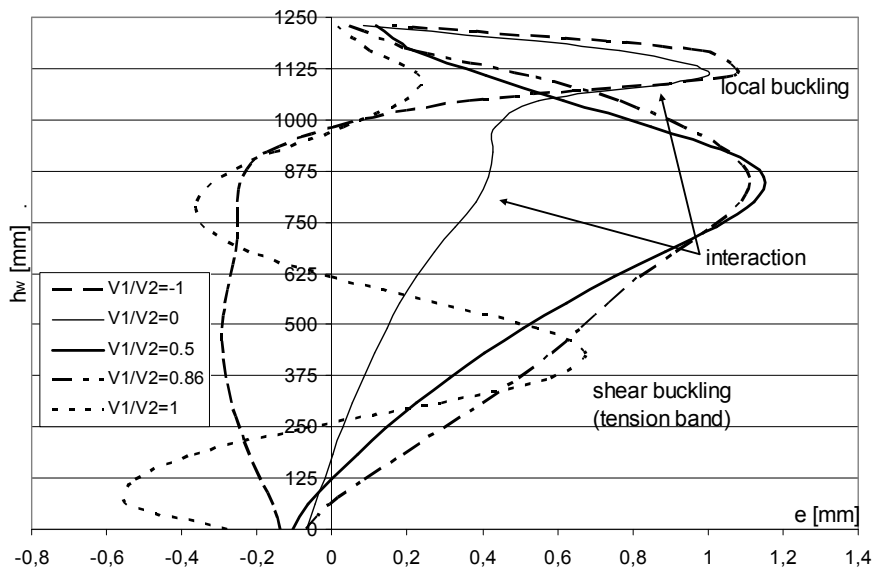

Fig. 13. Comparison of the lateral displacements - parallel fold.

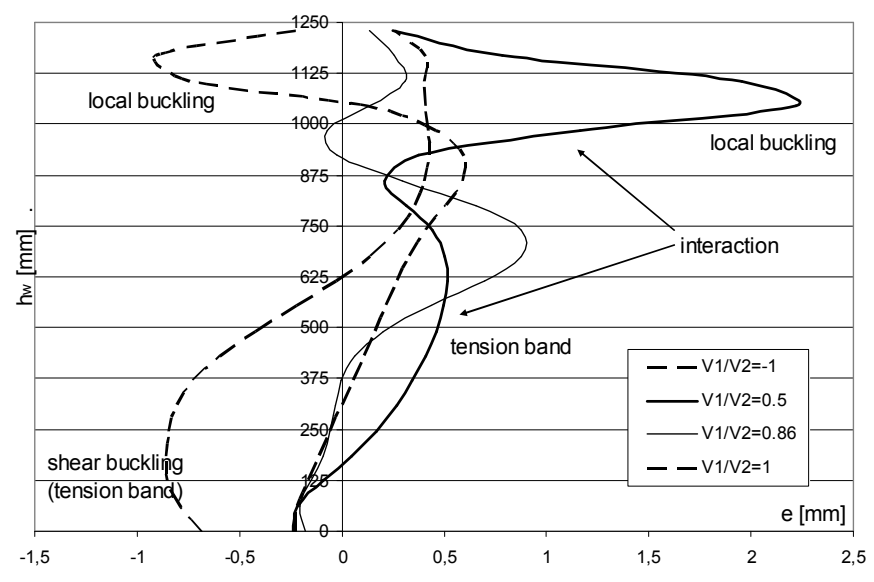

Fig. 14. Comparison of the lateral displacements - inclined fold.

\section{Evaluation of the results - development of interac- tion curves}

\subsection{Development of interaction curve}

In frame of the numerical parametric study the interaction between shear and patch loading is investigated. In the case of all analyzed girder geometry the ratio of $F$ and $V_{1}$ is changed. At first the pure patch loading and shear buckling resistances are determined and the observed failure modes are studied. After it 7-8 calculations are executed with different $F$ and $V_{1}$ ratios for each geometry. On this way about 10 calculations are conducted in average for each girder geometry, and in total 370 calculations are executed.

The results together with Elgaaly's numerical calculations are presented in two diagrams. The evaluation of the results is implemented by two different ways. The first (Fig. 15) is using the above mentioned method with subdivision of the combined loading situation according to Fig. 2 The horizontal axis of the diagram shows the transverse force divided by the pure patch loading resistance calculated by Ansys. The vertical axis shows the subdivided shear force divided by the shear buckling resistance of the web panel calculated by Ansys as well.

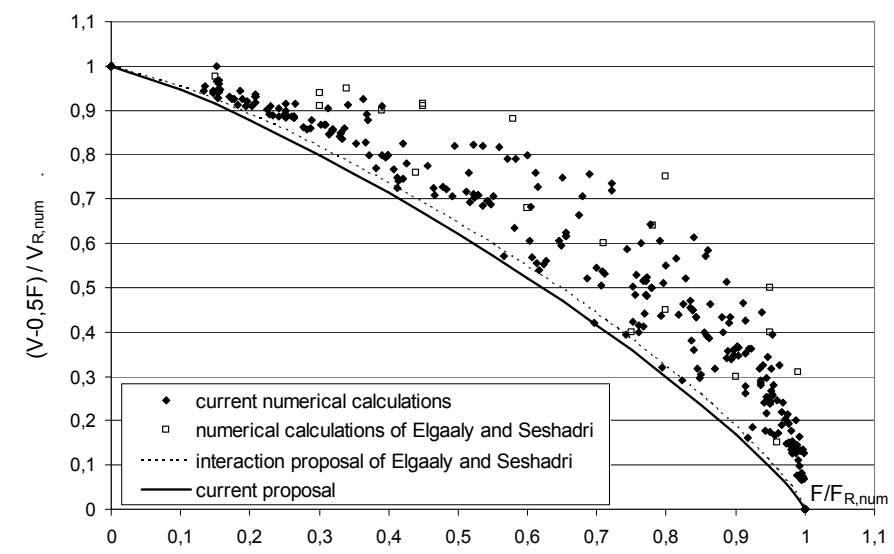

Fig. 15. Interaction of shear and patch loading - evaluation method I.

Fig. 15 shows that the recommended interaction curve of Elgaaly and Seshadri gives a good approximation in the extended parameter range. From all the numerical calculations only 3 results are under this interaction curve and all of them are at the edges of the analyzed parameter range (long loading length; large $\alpha$ value and large $a_{1}$ at the same time). According to take these special cases into account, a lower limit interaction curve is proposed in form of Eq. 2.

$$
\left(\frac{V-0.5 \cdot F}{V_{R}}\right)^{1.2}+\left(\frac{F}{F_{R}}\right)^{1.2} \leq 1.0
$$

There is an other possible evaluation method if the load subdivision method according to Fig. 2 is not used. In this case the vertical axis of the diagram shows the maximum shear force $\left(V_{2}\right)$ divided by the shear buckling resistance. Results based on this evaluation method are presented in Fig. 16 . The shear force due to pure patch loading is not subtracted from the maximum shear force and therefore a significant interacting shear force is 
present in case of pure patch loading, too. Points on the diagram are significantly higher than in case of the first evaluation method. The developed interaction curve may be expressed by Eq. (3).

$$
\left(\frac{V_{\max }}{V_{R}}\right)^{2.5}+\left(\frac{F}{F_{R}}\right)^{4} \leq 1.0
$$

The interaction proposal of Eq. 3 can be also useful, but the general shear and patch loading interaction evaluation method is based on the load subdivision procedure.

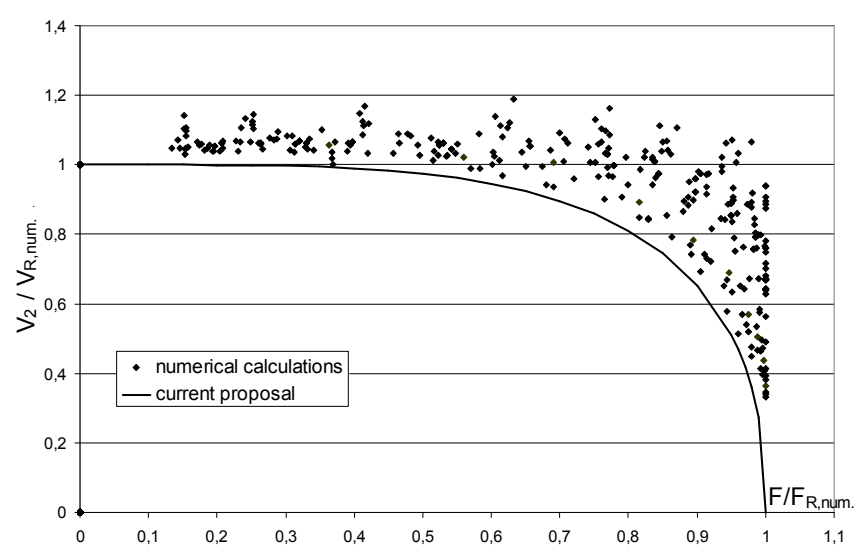

Fig. 16. Interaction between shear and patch loading - evaluation method II.

\subsection{Statistical evaluation of interaction curves}

In this chapter the discrepancy between interaction curves and numerical results are calculated. Vector lengths which goes from the zero point to each result are calculated $\left(r_{e}\right)$. The intersection of this vector and the analyzed interaction curve is calculated and the vector length of the intersection point is determined $\left(r_{t}\right)$. The ratio of the two lengths is called by $r$, which gives a good diagnostic about the distance of the calculated results and the interaction curve. The schematic overview of this calculation method can be seen in Fig. 17

$$
r=\frac{\overline{r_{e}}}{\overline{r_{t}}}
$$

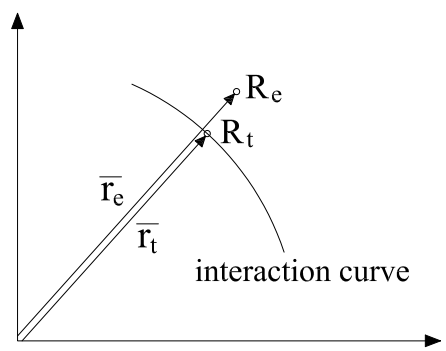

Fig. 17. Schematic overview of the calculation method.

Interaction curve can be interpreted on the way that both axis of the diagram are divided by the pure patch loading or shear buckling resistances which comes from experiments or numerical calculations. In this case the results of the numerical calculations are substituted in $V_{R}$ and $F_{R}$ as noted in Eq. (5). It leads to the analysis of the pure interaction behaviour which is independent from any design methods.

$$
\left(\frac{V-0.5 \cdot F}{V_{R, \text { num }}}\right)^{1.2}+\left(\frac{F}{F_{R, \text { num }}}\right)^{1.2} \leq 1.0
$$

The $r$ values are calculated for all numerical results, where pure patch loading and shear resistances are taken from the numerical calculations ( $V_{R, \text { num }}$ and $F_{R, \text { num }}$ ). The $r$ is also determined based on the interaction curve of Elgaaly and Seshadri [3] and based on the proposed interaction curve presented in this paper. Mean values, standard deviations, coefficient of variations and $5 \%$ fractiles and minimum and maximum values of $r$ are calculated and listed in Table 4 The calculation is executed based on the interaction curve of Elgaaly and Seshadri [3] and based on the currently proposed interaction curve.

Tab. 4. Statistical evaluation based on the resistances of $V_{R \_A n s y s}$

\begin{tabular}{ccc}
\hline & $\begin{array}{c}\text { Interaction curve of } \\
\text { Elgaaly and Seshadri }\end{array}$ & $\begin{array}{c}\text { Current interaction } \\
\text { proposal }\end{array}$ \\
\hline Mean value & 1.071 & 1.086 \\
\hline Standard deviation & 0.066 & 0.073 \\
\hline Coeff. of variation & 0.062 & 0.067 \\
\hline $5 \%$ lower fractile & 0.963 & 0.967 \\
\hline $5 \%$ upper fractile & 1.179 & 1.206 \\
\hline maximum & 1.350 & 1.381 \\
\hline minimum & 0.980 & 1.000 \\
\hline
\end{tabular}

Table 4 shows that the proposed interaction curve is near to the proposal of Elgaaly and Seshadri and gives slightly strong interaction criteria. The minimum value is equal to 1.0 therefore the currently proposed interaction curve can be applied for all girder geometry in the analyzed parameter range which is larger than the original one from Elgaaly and Seshadri [3]. Difference of the minimum and maximum values of $r$ is 1.38 , this means that the scatter of the results are relatively large. The value of the standard deviation proves also this observation.

\section{Conclusions}

In the framework of the presented research work the combined shear and patch loading of girders with corrugated steel web is analyzed. Previous experiments, numerical investigations and existing interaction curves are studied. Only one investigation is available in the literature dealing with this interaction type in case of corrugated web girders and it was focused only on a narrow parameter range and no experiments were executed. Therefore the focus of the current research is the determination of the shear and patch loading interaction behaviour of corrugated web girders by nonlinear Finite Element simulation for an extended parameter range.

Based on previous patch loading experiments conducted at the Budapest University of Technology and Economics a Finite 
Element model is developed and verified for all test specimens. The load carrying capacities are determined by geometrical and material nonlinear analysis using imperfections. Based on the calibrated numerical model a parametric study is executed to investigate the shear and patch loading interaction behaviour of corrugated web girders in a large parameter range. Results are compared to the previously developed interaction curves and a new design interaction equation is proposed which is applicable for corrugated web girders in the whole analyzed parameter range.

Statistical evaluation of both interaction curves are also executed and the applicability and safety level are determined. Comparison of the numerical results and interaction curves show that the two analyzed interaction curves are close to each other. The currently proposed interaction limit gives a slightly stronger interaction criteria and it can be applied in a wide parameter range.

\section{References}

1 KuhImann U, Braun B, Continued Shear- and Patch Loading: Numerical Studies and Development of an Interaction Equation, 2006. COMBRI project, RFCS Contract RFS-CR-03018, Document COMBRI-ReportUSTUTT-002.

2 EN 1993-1-5: 2006; Eurocode 3: Design of steel structures, Part 1-5: Plated structural elements, European Committee for Standardisation, Brussels, Belgium, 2006.

3 Elgaaly M, Seshadri A, Girders with corrugated webs under partial compressive edge loading, Journal of Structural Engineering (1997), no. 6, 783791.

4 ANSYS Release 10.0, Ansys Inc., 2007.

5 Leiva-Aravena L, Edlund B, Buckling of trapezoidally corrugated webs, ECCS Colloquium on Stability of Plates and Shells (Ghent University, Belgium, 1987), 1987.

6 Kähönen A, Zur Einleitung von Einzellasten in I-Träger mit trapezförmig profilierten Stegen loading, Stahlbau 57 (1988), no. 8, 250-252.

7 Luo R, Edlund B, Ultimate strength of girders with trapezoidally corrugated webs under patch loading, Thin-Walled Structures 123 (1996), no. 6, 135156.

8 Kövesdi B, Braun B, Kuhlmann U, Dunai L, Patch loading resistance of girders with corrugated webs, Journal of Constructional Steel Research 66 (2010), no. 12, 1445-1454.

9 Easley J T, McFarland D E, Buckling of light-gage corrugated metal shear diaphragms, Journal of the Structural Division, ASCE 95 (1996), no. 12, 1497-1516.

10 Elgaaly M, Hamilton R W, Seshadri A, Shear strength of beam with corrugated webs, Journal of Structural Engineering, ASCE 122 (1996), no. 4, 390-398.

11 Yamazaki M, Buckling strength of corrugated webs, Structural Engineering Research Papers, JSCE 47 (2001), 19-26.

12 Bergfelt A, Leiva L, Shear buckling of trapezoidally corrugated girder webs, Chalmers University of Technology, 1984.

13 Driver R G, Abbas H H, Sause R, Shear behaviour of corrugated web bridge girder, Journal of Structural Engineering, ASCE 132 (2006), no. 2, 195-203.

14 Scheer J, Tragverhalten von Biegeträgern mit trapezförmig profillierten Stegen und geschweissten Steg-Gurt-Verbindungen, Technical University Braunschweig. DFG Forschungsvorhaben, Ber. Nr. 6203/1.

15 Moon J, Jongwon Y, Byung $\mathbf{H} \mathbf{C h}$, Hak-Eun L, Shear strength and de- sign of trapezoidally corrugated steel webs, Journal of Constructional Steel Research 65 (2008), no. 5, 1198-1205.

16 Luo R, Edlund B, Shear capacity of plate girders with trapezoidally corrugated webs, Thin-Walled Structures 26 (1996), no. 1, 19-44.

17 Jongwon Y, Heungbae G, Kwangsoo Y, Hakeun L, Interactive shear buckling behaviour of trapezoidally corrugated steel webs, Engineering Structures 30 (2008), 1659-1666.

18 Kövesdi B, Dunai L, Determination of the patch loading resistance of girders with corrugated webs using nonlinear finite element analysis, Twelfth International Conference on Civil, Structural and Environmental Engineering Computing (Funchal, Portugal, 01.09.2009), 2009, pp. 18. ISBN:978-1905088-32-4. 